\title{
Hot Atmospheres Around Accreting Neutron Stars: A Possible Source For Hard X-ray Emission
}

\author{
Silvia Zane ${ }^{1}$ \\ International School for Advanced Studies, \\ Via Beirut 2-4, 34014 Trieste, Italy \\ e-mail: zane@sissa.it \\ Roberto Turolla \\ Dept. of Physics, University of Padova, \\ Via Marzolo 8, 35131 Padova, Italy \\ e-mail: turolla@astaxp.pd.infn.it \\ and \\ Aldo Treves ${ }^{2}$ \\ International School for Advanced Studies, \\ Via Beirut 2-4, 34014 Trieste, Italy \\ e-mail: treves@astmiu.uni.mi.astro.it
}

Received —_ accepted

\footnotetext{
${ }^{1}$ also at: Dept. of Physics, University of Illinois at Urbana-Champaign, 1110 W. Green St., Urbana, IL 61801-3080, USA

${ }^{2}$ present address: II Faculty of Sciences, University of Milano, Via Lucini 3, 22100, Como, Italy
} 


\begin{abstract}
The structure of static atmospheres around unmagnetized neutron stars undergoing steady, spherical accretion is discussed. We focus on the "hot" configurations presented by Turolla et al. (1994) and calculate the radiation spectrum using a characteristics method. In particular, it is found that $\mathrm{e}^{+}-\mathrm{e}^{-}$ pair production may affect significantly the external atmospheric layers, where positron and proton number densities become of the same order. The consequent increase of the scattering opacity lowers the Eddington limit and this, in turn, may drive a dynamical instability if the accretion luminosity is large enough, ultimately producing a rapid expulsion of the envelope. If "hot" states are indeed accessible, this mechanism could give rise to transient phenomena in hard X-rays of potential great astrophysical interest.
\end{abstract}

Subject headings: accretion, accretion disks — radiative transfer — stars: neutron - X-rays: general 


\section{Introduction}

The problem of calculating the radiation spectrum produced in the atmosphere of an unmagnetized, accreting neutron star (NS) received wide attention in the past, starting from the pioneering work by Zel'dovich, \& Shakura (1969, hereafter ZS) where the case of stationary, spherical accretion was first discussed. In the commonly accepted picture, steaming from ZS' original analysis and corroborated by the more detailed numerical work by Alme, \& Wilson (1973), the emerging spectrum is essentially a blackbody plus a high-energy Compton tail. It can be characterized by two parameters, the accretion

luminosity $L_{\infty}$ (or, equivalently, the accretion rate) and the column density $y_{0}$ corresponding to the penetration range of incoming protons in the NS atmosphere. Recently Zampieri et al. (1995, paper II) extended ZS's results to cover the low luminosities typical of old, isolated NSs accreting the interstellar medium $\left(L \lesssim 10^{34} \mathrm{erg} / \mathrm{s}\right)$, showing that deviations from a blackbody become more conspicuous as the luminosity decreases.

Turolla et al. (1994, paper I) re-examined the issue of the thermal and radiative properties of static atmospheres around accreting NSs and pointed out that a new class of much hotter equilibrium solutions may exist if the accretion luminosity exceeds a certain critical value, in analogy with what has been already found in black hole accretion (see e.g. Park 1990; Nobili, Turolla, \& Zampieri 1991). The existence of two states is related to the different role thermal and non-thermal processes may play in exchanging energy between photons and electrons in the atmosphere: in the "cold" state (à la ZS) the energy released by accretion is radiated away essentially via bremsstrahlung, whereas in the "hot" state Compton scattering dominates (in this respect see also Burger, \& Katz 1980).

In paper I "hot" solutions were found solving the frequency-integrated transfer problem. The lack of any information about the angular and frequency dependence of the radiation field made impossible to evaluate the photon-photon pair production, which was 
nevertheless recognized to be potentially important, and led to introduce rather drastic assumptions in the calculation of the Compton energy exchange rate.

In this paper we try to overcome some of these limitations, in the attempt to shed light on the relevance of "hot" solutions. To get some physical insight without resorting to a full time-dependent calculation, we consider the stationary case and analyze the effects of different processes separately. First the radiation field is calculated using the characteristics method introduced by Zane et al. (1996, paper III). Pair production is neglected and the frequency-dependent transfer equation is solved coupled to the hydrostatic and energy balance. The resulting intensity is then used to estimate the pair number density $n_{+}$ produced by photon-photon interactions. Although not fully self-consistent, this approach shows that a large pair production is expected in the external atmospheric layers. This opens the possibility that such configurations are unstable with respect to the onset of a radiatively-driven wind, produced by the increased opacity in the pair-rich plasma. Astrophysical applications of "hot" atmospheres are suggested in the context of hard X-ray transient phenomena.

\section{The Model}

In this section we briefly outline the input physics of our model, referring to previous

works (ZS; Alme, \& Wilson 1973; paper I and II) for all details, and introduce our radiative transfer calculations.

We consider an unmagnetized, non-rotating neutron star with mass $M_{*}=1 M_{\odot}$ and radius $R_{*}=10^{6} \mathrm{~cm}$, surrounded by a static atmosphere where the accreting flow is decelerated by Coulomb collisions and/or plasma interactions. As the flow hits the outermost stellar layers, the proton bulk kinetic energy is converted into electron thermal 
energy and then re-emitted as electromagnetic radiation. The heat injected per unit time and mass, $W_{h}$, is given by (see Bildsten, Salpeter, \& Wasserman 1992; paper II)

$$
W_{h}=\frac{L_{\infty}}{8 \pi R_{*}^{2} y_{0} y_{g}} \frac{1+v_{t h}^{2} / v_{i}^{2}}{\left[1-\left(1-v_{t h}^{4} / v_{i}^{4}\right)\left(y / y_{0}\right)\right]^{1 / 2}}
$$

where $y_{g}=\left(1-R_{g} / R_{*}\right)^{1 / 2}$ is the gravitational redshift factor, $R_{g}=2 G M_{*} / c^{2}$, $v_{t h}^{2}=3 K T / m_{p}, T$ is the gas temperature, $v_{i}^{2}=c^{2}\left(1-L_{\infty} / L_{E}\right)\left(R_{g} / R_{*}\right), y=\int_{R}^{\infty} \rho d R$ is the column density and $L_{E}$ is the Eddington luminosity. As discussed in paper I, these solutions show no significant envelope expansion, so the radial coordinate can be safely assumed equal to the star radius. The atmospheric structure can be solved in plane-parallel geometry, using as independent variable the scattering optical depth $\tau=\kappa_{e s} y$, where $\kappa_{e s}$ is the Thomson opacity. In this case, the luminosity profile can be immediately derived from the zero-th frequency-integrated moment equation (see paper II). The run of pressure $P$ and temperature $T$ are given by the solution of the hydrostatic and energy balance equations (see again paper I and II)

$$
\begin{gathered}
\frac{d P}{d \tau}=\frac{G M_{*}}{y_{g}^{2} R_{*}^{2} \kappa_{e s}}\left(1-y_{g} \frac{\kappa_{1}}{\kappa_{e s}} \frac{L}{L_{E}}\right), \\
\frac{W}{c}=\kappa_{P}\left(a T^{4}-\frac{\kappa_{0}}{\kappa_{P}} U\right)+(\Gamma-\Lambda)_{C},
\end{gathered}
$$

with the boundary condition of vanishing pressure at the top of the atmosphere. In equation (3) $U$ is the radiation energy density, $\kappa_{P}, \kappa_{0}$ and $\kappa_{1}$ are the Planck, absorption, and flux mean opacities and $(\Gamma-\Lambda)_{C}$ is the Compton energy exchange rate. The effective heating $W$ represents the fraction of $W_{h}$ which is converted into electromagnetic radiation within the atmosphere; $W$ coincides with $W_{h}$ if no energy is injected at the inner boundary, that is if $L_{i n}=0$. Since at large depths the radiation field becomes more and more isotropic, $L_{i n}$ is indeed small, although in a realistic situation it is not exactly zero. On the other hand, the thermal structure of both the "cold" and "hot" solutions presented in paper I proved not sensitive to the assumed value of the luminosity at the bottom of the atmosphere, provided 
it is not too large compared with $L_{\infty}$. In particular, "hot" atmospheres exhibit a rather sharp drop in the gas temperature which separates two distinct regions characterized by different thermal properties. In the external layers, where the bulk of the luminosity is produced, the energy released by accretion is radiated away via Compton cooling, while in the deeper, denser layers LTE is attained and the temperature profile is mainly determined by bremsstrahlung equilibrium. For these solutions $L_{i n}$ also coincides with the value of the radiative flux at the top of the cold region.

There are two important points concerning the "hot" models that were not thoroughly discussed in paper I and deserves further analysis. Both of them concern the possibility of establishing and maintaining a rather steep temperature gradient between the hot to the cold part of the atmosphere, so that a "hot" solution can indeed set in. First of all we note that the position of the temperature drop is practically unrelated the value of the proton stopping length. Although for $y_{0}=20$ (the value we will use here) they practically coincide, we stress that, in general, the value of the scattering depth at which the transition occurs depends only on the relative efficiency of Compton and bremsstrahlung heating-cooling and, as numerical tests show, it is always close to $\tau_{t r}=10\left(y_{t r}=25\right)$. The presence of a sudden decrease in $T$ can be understood comparing the free-free and Compton thermal times

$$
\frac{t_{C}}{t_{f f}}=\frac{\epsilon_{f f}\left(1-U / a T^{4}\right)}{4 k\left|T-T_{\gamma}\right| \kappa_{e s} U / m_{e} c^{2}},
$$

where $\epsilon_{f f}$ is the free-free emission and $T_{\gamma}$ is the radiation temperature. As the scattering depth increases $T$ and $T_{\gamma}$ become closer, because non-conservative scatterings tend to establish thermal equilibrium, and $U$ either increases or stays constant. In the hot, effectively thin layers $U \ll a T^{4}$ and at large enough $\tau$, where $T \simeq T_{\gamma}$, the time scale for free-free cooling becomes shorter than the Compton time. The plasma temperature drops until $a T^{4} \simeq U$ in order for the energy balance to be satisfied and the radiation temperature follows. 
The second important point concerns the efficiency of electron conduction at the interface between the hot and cold regions. This effect was not considered in paper I. In order to get a quantitative estimate of the relevance of thermoconduction we compare the energy flux due to conduction, $F_{c}=\nu_{c} \rho d T / d y$, with the radiative flux, $F_{r}=L / 4 \pi R^{2}$. Using the expression for $\nu_{c}$ given in Zel'dovich, \& Raizer (1967) it is

$$
\frac{F_{c}}{F_{r}} \sim 2.5 \times 10^{-3}\left(\frac{T}{10^{9}}\right)^{5 / 2}\left(\frac{L}{L_{E}}\right)^{-1} \frac{y_{t r}}{\Delta y}
$$

where $\Delta y$ is the width of the transition layer and $T$ is the temperature in the hot region. As the previous equation shows, electron conduction is indeed efficient in transferring heat from the hot to the cold region. However, if a fraction of the luminosity is produced below $y_{t r}, L_{i n} / L_{\infty} \sim 0.2-0.3$ for $L_{\infty} \sim 0.1 L_{E}$, conduction will limit the temperature of the hot region to $\sim 10^{9} \mathrm{~K}$, smear the jump over $\Delta y \sim 2-3$ and then cease to be important. The stationary temperature profile will then look quite similar to that discussed by Zel'dovich, \& Raizer (1967) in the context of shock waves in a plasma. Although the real profile will deviate from that of paper I (mainly in the absence of the peak at $T \sim 10^{10} \mathrm{~K}$, see curve $\mathrm{b}$ in figure 2, which will be leveled if conduction is taken into account), the emerging picture seems to be substantially unaltered by the inclusion of thermal conduction. This conclusion is reinforced by the result of the frequency-dependent calculation presented here which shows that the temperature profile is nearly isothermal at $T \sim 10^{9} \mathrm{~K}$ (see curve a in figure 2 ). It is interesting to point out that the existence of a "hot" equilibrium state can be recovered analytically by solving the energy balance equation in the limit in which Compton cooling dominates and using an approximated expression for the Comptonized radiation spectrum (see Titarchuk 1997, Titarchuk, Lapidus \& Muslimov 1997). In the case in which Comptonization is effective and produces a rather flat spectrum with $\alpha \ll 1$ and the scattering depth at the stopping radius exceeds unity it is $T \sim 10^{9} \mathrm{~K}$ quite independently on the value of $y_{0}$ (Titarchuk, private communication). 
In the following we consider in more detail "hot" atmospheres. As we discussed, in this picture only a small fraction of the total luminosity $\left(L_{i n} / L_{\infty} \lesssim 0.2-0.3\right)$ is produced below $y_{t r}$, where the scattering depth is already larger than $\sim 10$. Bearing this in mind, we can compute the radiation field only for $y<y_{t r}$ with a suitable boundary condition at $y_{t r}$. The consistency of the computed flux at the boundary with the assumed value of $L_{i n}$ will be checked a posteriori. Radiative transfer is solved by means of the characteristics method discussed in paper III and yields the full spatial, angular and frequency dependence of the photon occupation number $f=c^{2} I / 2 h^{4} \nu^{3}$, where $I$ is the specific intensity. In the case of a static, plane-parallel atmosphere $f$ depends on one "spatial" variable, $\tau$, on the cosine of the angle between the photon propagation direction and the vertical direction, $\mu$, and on the local photon energy $E=h \nu$. The spectrum observed at infinity is obtained redshifting to energies $E_{\infty}=E\left(1-R_{g} / R_{*}\right)^{1 / 2}$ that at the top of the atmosphere. We used as boundary conditions $f=0$ at the top of the atmosphere for incoming rays and a regularity condition $(d f / d \tau=0)$ at the inner boundary for outgoing rays. The interested reader is referred again to paper III for all computational details about the source term which includes relativistic e- $\mathrm{p}$, e-e bremsstrahlung and Compton scattering. In particular, Compton scattering is treated in its more general form by direct evaluation of the integrals of the Compton scattering kernel. To avoid numerical instabilities which arise when more accurate formulae are used (see e.g. Shestakov, Kershaw, \& Prasad 1988), the Kompaneets approximation was retained in the calculation of the Compton energy exchange rate.

The resulting monochromatic energy density is shown in figure 1 for $L_{\infty} / L_{E}=7 \times 10^{-2}$, $L_{i n}=0.2 L_{\infty}$ and $y_{0}=20 \mathrm{~g} \mathrm{~cm}^{-2}$ at different optical depths. The dashed line represents the spectrum observed at infinity, i.e. corrected for the gravitational redshift. This model has been computed solving the transfer equation for 20 values of $\mu, 25$ energies in the range $0.017 \mathrm{MeV} \leq E \leq 5 \mathrm{MeV}$ and $10^{-3} \leq \tau \leq 8$. The considered luminosity is close to the lower limit for the existence of "hot" solutions with $y_{0}=20 \mathrm{~g} \mathrm{~cm}^{-2}$, and it can be thought to be 
well representative of situations where photon energies are large enough to produce pairs, since $T_{\gamma}$ is anticorrelated with $L_{\infty}$ (see paper I). The emergent flux, in fact, is peaked at $\sim 500 \mathrm{keV}$ and is characterized by a high-energy tail. The gas and radiation temperatures, together with the correspondent profiles derived in paper I, are shown in figure 2. The lower values of $T$ (about a factor 3 ) are mainly due to the different calculation of $T_{\gamma}$ which enters in the energy equation. Now the radiation temperature is directly evaluated from the mean intensity and not obtained from a phenomenological equation, as in paper I. In the present calculation, the atmosphere appears to be nearly isothermal. In both cases the energy balance is practically obtained equating Compton heating and cooling, which gives $T \sim T_{\gamma}$. Now $T$ is always close to $10^{9} \mathrm{~K}$ and the computed luminosity at the inner boundary is $\sim 0.02 L_{E} \sim 30 \% L_{\infty}$ (quite close to the input value), so conduction is not expected to be much effective.

\section{Pair Processes}

We use the results derived in the previous section to estimate the pair number density $n_{+}$, under the simplifying hypothesis that pairs thermalize with electrons and protons in the atmosphere. Clearly this is just an ad hoc assumption that needs to be checked a posteriori (see the discussion at the end of the section).

Let us consider the process

$$
\gamma+\gamma \rightleftharpoons e^{+}+e^{-}
$$

quantities relative to the two photons will be denoted with indices 1,2 . The absorption coefficient for a photon of dimensionless energy $x_{1}=h \nu_{1} / m_{e} c^{2}$ propagating in direction $\mu_{1}$ through a radiation field of intensity $I_{2}=I\left(\tau, x_{2}, \mu_{2}\right)$ is given by

$$
a_{\gamma \gamma}\left(x_{1}\right)=\frac{1}{h c} \int \frac{I_{2}}{x_{2}} \sigma(x)(1-\mu) d x_{2} d \mu_{2} d \phi_{2},
$$


where $x^{2}=x_{1} x_{2}(1-\mu) / 2$ is the square of the photon energy in the center-ofmomentum frame, $\mu$ is the cosine of the angle between the two photon directions, $\phi_{2}$ is the azimuthal angle and $\sigma(x)$ is the photon-photon pair production cross-section (see e.g. Jauch, \& Rohrlich 1976). Replacing $\phi_{2}$ with $\phi=\phi_{2}-\phi_{1}$ and introducing $x_{ \pm}^{2}=x_{1} x_{2}\left[1-\cos \left(\theta_{1} \pm \theta_{2}\right)\right] / 2$ (see Stepney, \& Guilbert 1983), expression (7) becomes

$$
a_{\gamma \gamma}=\frac{4}{h c} \int d x_{2} d \mu_{2} \frac{I_{2}}{x_{1} x_{2}^{2}} F\left(x_{+}, x_{-}\right) .
$$

The function

$$
F\left(x_{+}, x_{-}\right)=\int_{x_{-}}^{x_{+}} \frac{\sigma(x) x^{3} d x}{\sqrt{\left(x_{+}^{2}-x^{2}\right)\left(x^{2}-x_{-}^{2}\right)}}
$$

does not depend on the specific intensity so it was calculated once for all on a fixed grid of values of $x_{ \pm}$and stored in a matrix. The outer double integral was then computed using a spline interpolation. Once the absorption coefficient is known, the pair production rate $R_{\gamma \gamma}$ is obtained performing two further integrations over angles and energies

$$
R_{\gamma \gamma}=\frac{\pi}{h} \int d x_{1} d \mu_{1} \frac{I_{1}}{x_{1}} a_{\gamma \gamma}
$$

Following Svensson (1982) and introducing $\theta=K T / m_{e} c^{2}$, the pair annihilation rate can be expressed in terms of a dimensionless function $A(\theta)$ as

$$
\dot{n}_{+}=n_{+} n_{-} c r_{e}^{2} A(\theta)
$$

where

$$
A(\theta) \simeq \frac{\pi}{1+2 \theta^{2} / \ln (2 \eta \theta)}
$$

$\eta \simeq 0.56146$ and $r_{e}$ is the classical electron radius. Combining the previous expressions, the pair balance equation becomes in the stationary case

$$
R_{\gamma \gamma}-z(1+z) n^{2} c r_{e}^{2} A(\theta)=0
$$


where $z=n_{+} / n, n$ is the proton number density and the charge neutrality condition $n_{-}=n_{+}+n$ has been used. The positive root of equation (13) is

$$
z(\tau)=\frac{1}{4}\left(\sqrt{1+\frac{16 R_{\gamma \gamma}}{n^{2} A(\theta) r_{e}^{2} c^{2}}}-1\right) .
$$

Using the specific intensity and the run of thermodynamical variables derived in section 2 , we calculated the proton and positron number densities shown in figure 3 , together with $z$. As it can be seen, while in the inner atmospheric regions no relevant pair production is expected, the value of $z$ becomes $\sim 10$ for $\tau \approx 10^{-3}$ and tends to increase in the external regions.

Since the Eddington limit in a pair plasma is lowered by a factor $m_{e} / m_{p}$, there is the possibility that large values of $z$ are never reached, because pairs are accelerated outwards by the radiative force as soon as they are created. The characteristic timescale for radiative acceleration $t_{a c c} \sim(2 h / a)^{1 / 2} \sim 5 \times 10^{-9}(h / 10)^{1 / 2}\left(L_{\infty} / L_{E}\right)^{-1 / 2} \mathrm{~s}, h \sim 10 \mathrm{~cm}$ is the scale height of the envelope and $a \sim\left(G M / R_{*}\right)\left(L / L_{E}\right)$ is the radiative acceleration, should be compared with the timescales for particle collisions and for the development of plasma instabilities. Coulomb collisions establish thermal equilibrium in a time $t_{\text {coll }} \sim 6 \times 10^{-7}\left(T / 10^{9}\right)^{3 / 2}\left(n / 10^{18}\right)^{-1} \mathrm{~s}$, while the growth time for the counter-streaming instability is $t_{\text {ins }} \sim 2 \times 10^{-11}\left(n / 10^{18}\right)^{-1 / 2} \mathrm{~s}$ (see e.g. Melrose 1986). Plasma instabilities have therefore enough time to produce microturbulence which, in turn, will efficiently couple $e^{ \pm}$ to the ambient plasma. Enhanced particle scattering prevents pairs from escaping and also allows them to thermalize with atmospheric electrons. Our initial assumption that pair production/annihilation is in equilibrium seems indeed justified. 


\section{Discussion and Conclusions}

The existence of "hot" solutions, first proposed in paper I, has been confirmed by means of a more detailed calculation of the radiation field. Our frequency- and angle-dependent approach allowed us to compute the specific intensity and hence to evaluate the photon-photon pair production. The temperature and density profiles are close to those presented in paper I and the spectrum peaks at about $500 \mathrm{keV}$.

First of all, we wish to comment about the possibility of getting a "hot" solution started. Of course, the thermal energy stored in a "hot" atmosphere is much larger than in a "cold" one, and it is roughly

$$
U_{t h} \sim 4 \pi R_{*}^{2} y_{t r}\left(\frac{K T}{m_{p}}\right),
$$

where $T$ is the average temperature. Assuming $K T \sim 100 \mathrm{keV}, U_{t h} \sim 10^{31} \mathrm{erg}$, a factor $\sim 100$ above the "cold" case. The transfer of energy from protons to electrons in our model is described by equation (1). Obviously, this is a rather crude estimate which does not enter into the details of the physical processes responsible for the energy exchange. Heating associated with the proton stopping might produce temperatures only in the keV range, so the transition between "cold" and "hot" states could require some more efficient heating process, like dissipation of shock waves or magnetic field reconnection. We note, however, that the proton bulk kinetic energy at the neutron star surface is $\sim 100 \mathrm{MeV}$, which is much higher than the temperature of "hot" solutions. In any case, the smallness of the energy content of the atmosphere compared to the value of the luminosity, $L_{\infty} \sim 10^{37} \mathrm{erg} / \mathrm{s}$, strongly indicates that there should be no severe physical hindrance to drive the transition on time scales of microseconds.

On the other hand, one can argue that the "hot" state should be short-lived. In fact, as shown is section 3, pair production is expected to become important, at least in the outer 
layers where the pair density reaches its equilibrium value at $z \sim 1-10$. Due to the extra opacity produced by $e^{ \pm}$, the critical luminosity in the atmosphere becomes a factor $1+2 z$ lower than the Eddington limit. In "hot" models with $L_{\infty} \sim 0.1 L_{E}$, where pairs appear to be coupled with the plasma, part of the envelope will become necessary dynamically unstable when $z \sim 5$. This conclusion probably remains valid even if the appearance of pairs alters the temperature profile and in a complete self-consistent solution the value of $z$ is lower than that considered here. One should expect the outer layers to be expelled very quickly at the onset of the "hot" state: as a consequence accretion may be inhibited with the possible production of a relativistic shock wave. This suggestion is corroborated by the consideration that the momentum fluxes of the two flows are within an order of magnitude, as can be seen comparing $\dot{M}_{a c c} v_{\text {acc }}$ and $\dot{M}_{\text {out }} v_{\text {out }} \approx M_{\text {out }} a$, where $M_{\text {out }} \sim 4 \pi R_{*}^{2} y$ is the mass in the unstable part of the atmosphere and $a \sim G M\left[(1+2 z) L / L_{E}-1\right] / R_{*}^{2}$ is the acceleration. Using the numerical results of section 3 and assuming $\dot{M}_{a c c} \sim L\left(R_{*} / G M\right)$, $v_{a c c}=c\left(R_{g} / R_{*}\right)^{1 / 2}$, which gives an upper limit for the momentum flux of the ingoing material, it turns out that $\dot{M}_{a c c} v_{\text {acc }} \lesssim 10 \dot{M}_{\text {out }} v_{\text {out }}$. The decrease of the accretion rate and of the luminosity may push the system in a regime where only the "cold" solution exists, giving rise to an on-off behaviour.

The "hot" state should be characterized by a spectrum close to that shown in figure 1 , with typical emission at $\approx 100 \mathrm{keV}$. Moreover, pair bursts are expected, each consisting of $\sim 4 \pi R_{*}^{2} y z / m_{p} \sim 6 \times 10^{35}$ particles; an upper limit for the integrated luminosity in the annihilation line will be of order of $10^{29} \mathrm{erg}$. These hot, optically thin and Comptonized envelopes bear some resemblance with hot coronae above accretion disks, although the geometry, the heating process, and the triggering of the pair instability may be different. The onset of the "hot" state represents a possible physical mechanism for producing efficiently high energy radiation from weakly magnetized, accreting neutron stars and may be of interest in connection with hard $\mathrm{X}$-ray transients, although we are aware that some 
simplifying assumptions, like spherical symmetry and absence of magnetic fields, may limit the applicability of our results.

At present hard emission $(E \gtrsim 30 \mathrm{keV})$ has been observed from a number of sources, believed to contain NSs, with SIGMA and BATSE (see e.g. Tavani, \& Liang 1996; Vargas et al. 1996). At least seven X-ray bursters, including Aql X-1, and other LMXBs show transient emission in the range $30-200 \mathrm{keV}$, with intensity anticorrelated with that in the soft band. These observations are of the utmost importance, since hard X-rays emission were previously associated only with $\mathrm{X}$-ray pulsators and black hole candidates. Tavani, \& Liang have proposed a possible explanation for the transient, hard state of these sources in terms of magnetic reconnection in the inner regions of an accretion disc around the NS, but the nature of the primary emission mechanism is still an open issue. The total X-ray luminosity of these sources is $\sim 10^{36}-10^{37} \mathrm{erg} / \mathrm{s}$, close to that considered in our model, although the duration of the hard state is several days. In our picture, this would imply that the appearance of $\mathrm{e}^{ \pm}$pairs, even if it affects the accretion process, does not induce the transition to the "cold" state. Hard states of shorter duration would be impossible to detect with BATSE and SIGMA, but they may be observed by the instrumentation on board of XTE or SAX.

We are indebted both to an anonymous referee for drawing our attention on the importance of thermal conduction in "hot" models and to the second referee, Lev Titarchuk, for his clarifying comments. The revised version of this paper greatly benefited from discussions with Fred Lamb, Luciano Nobili and Luca Zampieri. We are also grateful to Marco Tavani for useful discussions about hard X-ray transients. 


\section{REFERENCES}

Alme, M.L., \& Wilson, J.R. 1973, ApJ, 186, 1015

Burger, H.L., \& Katz, J.I. 1980, ApJ, 236, 921

Bildsten, L., Salpeter, E.E, \& Wasserman, I. 1992, ApJ, 384, 143

Jauch, J.M., \& Rohrlich, F. 1976, The Theory of Photons and Electrons (New York: Springer-Verlag)

Melrose, D.B. 1986, Instabilities in Space and Laboratory Plasmas (Cambridge: Cambridge University Press)

Nobili, L., Turolla, R., \& Zampieri, L. 1991, ApJ, 383, 250

Park, M-G. 1990, ApJ, 354, 64

Shestakov, A.I., Kershaw, D.S., \& Prasad, M.K. 1988, J. Quantit. Spectros. Radiat. Transfer, 40, 577

Stepney, S., \& Guilbert, P.W. 1983, MNRAS, 204, 1269

Svensson, R., 1982, ApJ, 258, 335

Tavani, M., \& Liang, E. 1996, A\&A, in press

Titarchuk, L. 1997, in proceedings of the 2nd Integral Workshop, ESA SP-382

Titarchuk, L, Lapidus, I., \& Muslimov, A. 1997, ApJ, submitted

Turolla, R., Zampieri, L., Colpi, M., \& Treves, A. 1994, ApJ, 426, L35 (paper I)

Vargas, M., et al. 1996, A\&A, 313, 828

Zampieri, L., Turolla R., Zane S., \& Treves, A. 1995, ApJ, 439, 849 (paper II)

Zane S., Turolla, R., Nobili, L., \& Erna, M. 1996, ApJ, 466, 871 (paper III)

Zel'dovich, Ya, \& Raizer, Yu. 1967, Physics of Shock Waves and High-Temperature Hydrodynamic Phenomena (New York: Academic Press) 
Zel'dovich, Ya., \& Shakura, N. 1969, Soviet Astron.-AJ, 13, 175 (ZS) 
Fig. 1.- Monochromatic mean intensity for the model $L_{\infty}=7 \times 10^{-2} L_{E}, y_{0}=20 \mathrm{~g} \mathrm{~cm}^{-2}$; different lines correspond to equally spaced values of $\log \tau$ in the interval $[-3,0.9]$. The emerging redshifted spectrum is also shown (dashed line).

Fig. 2.- a) The gas temperature (full line) and the radiation temperature (dashed line) for the model of figure 1. b) Same results from the frequency-integrated analysis of paper I.

Fig. 3.- Proton (full line), positron (dashed line) number densities (in units of $10^{22} \mathrm{~cm}^{-3}$ ) and $z=n_{+} / n$ (dash-dotted line) versus the scattering optical depth for the model of figure 1. 


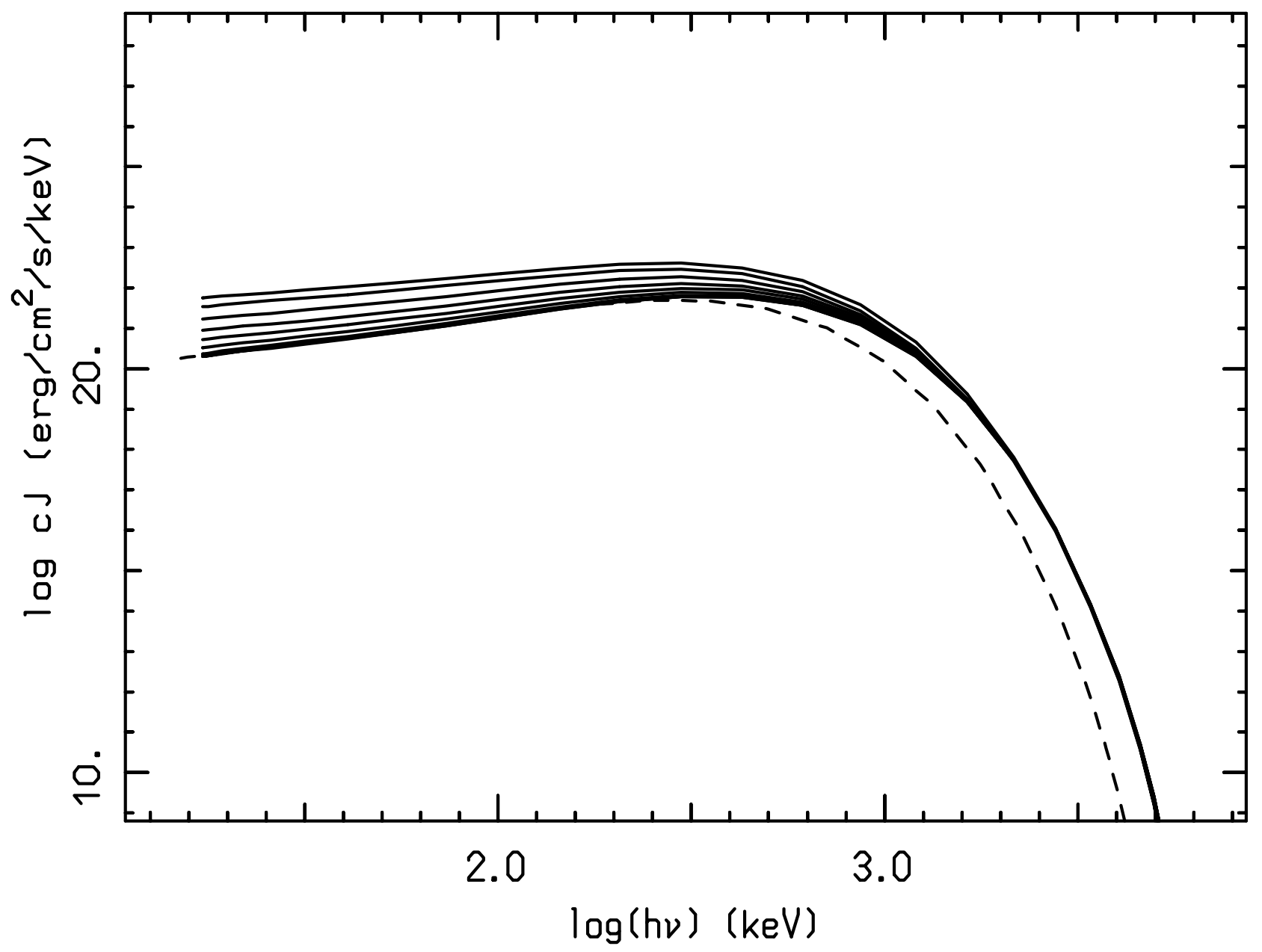




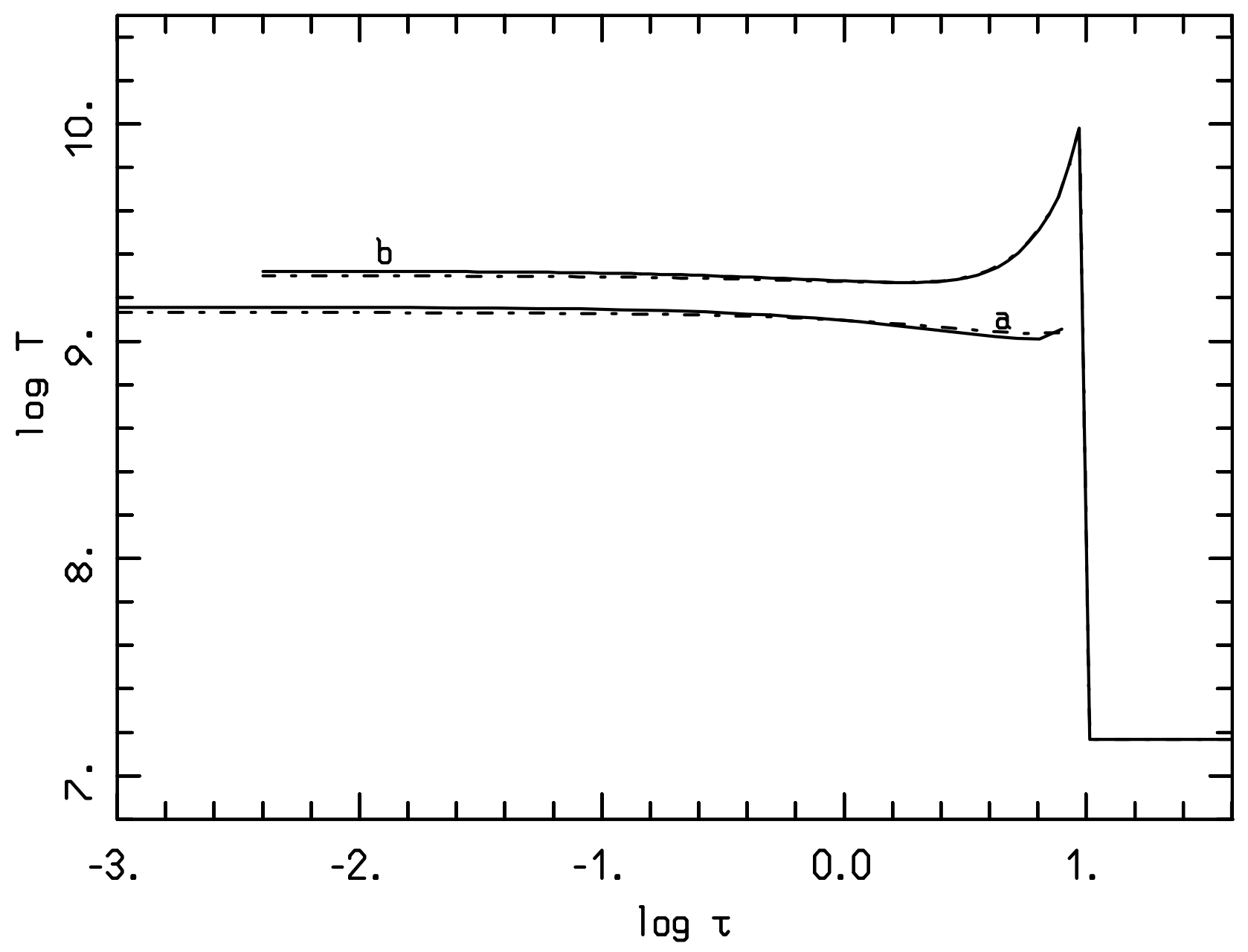




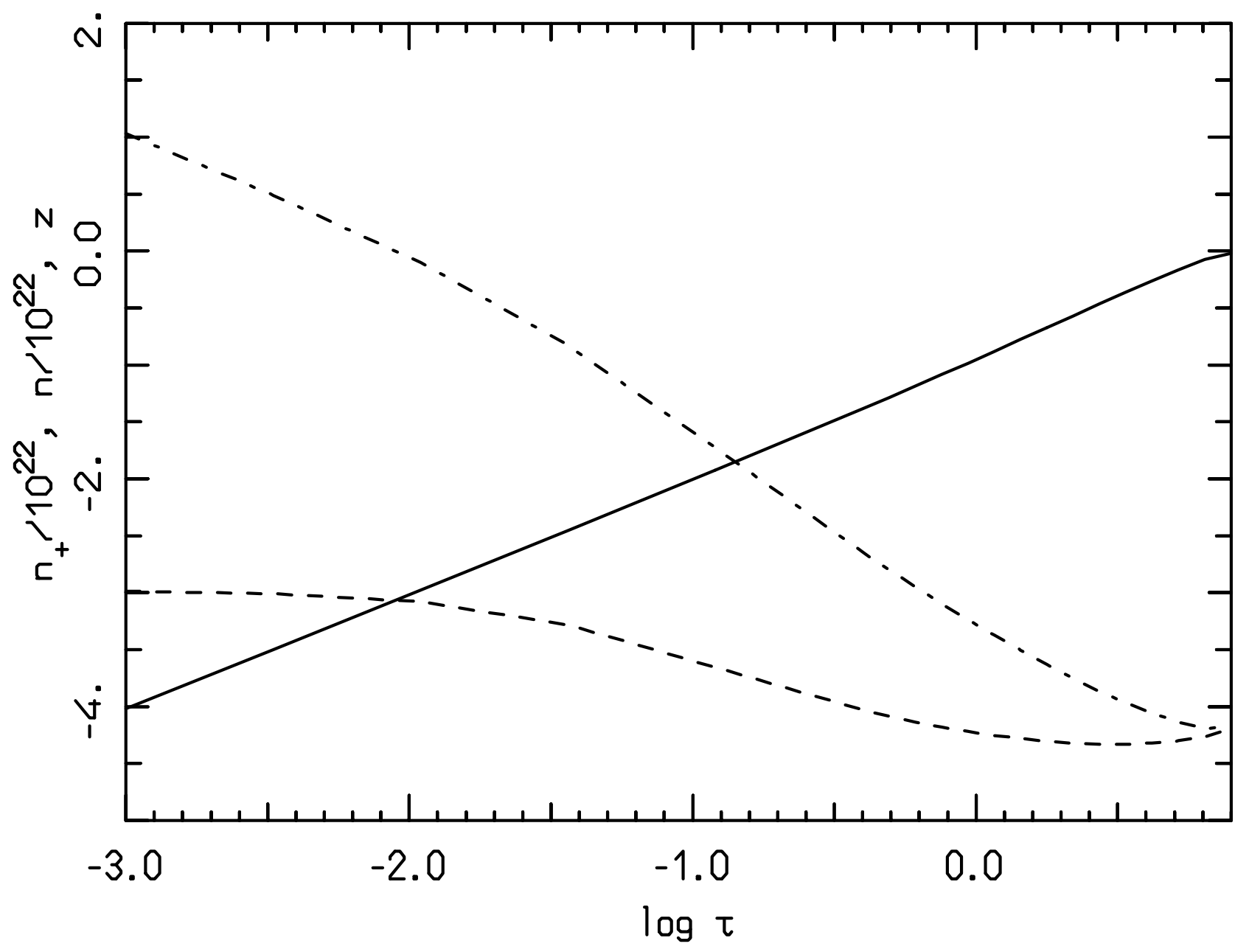

